\title{
Protective Effects of Crocetin against Radiation-Induced Injury in Intestinal Epithelial Cells
}

\author{
Chen Zhang, ${ }^{1,2}$ Kequan Chen, ${ }^{3}$ Jinghua Wang, ${ }^{4}$ Zhongwen Zheng, ${ }^{2}$ Yujun Luo, ${ }^{2}$ \\ Weijie Zhou, ${ }^{2}$ Zewei Zhuo, ${ }^{2}$ Jun Liang $\mathbb{D}^{5},{ }^{5}$ Weihong Sha $\mathbb{D}^{1,2}$ and Hao Chen ${ }^{10}{ }^{2}$ \\ ${ }^{1}$ Department of Gastroenterology, Affiliated South China Hospital, Southern Medical University (Guangdong Provincial \\ People's Hospital), Guangzhou 510080, China \\ ${ }^{2}$ Department of Gastroenterology, Guangdong Provincial People’s Hospital, Guangdong Academy of Medical Sciences, \\ Guangzhou 510080, China \\ ${ }^{3}$ Department of Gastroenterology, The First Affiliated Hospital of Guangzhou Medical University, Guangzhou 510120, China \\ ${ }^{4}$ Department of Hematology, Guangdong Provincial People's Hospital, Guangdong Academy of Medical Sciences, \\ Guangzhou 510080, China \\ ${ }^{5}$ Department of Critical Care Medicine, Guangdong Provincial Geriatrics Institute, Guangdong Provincial People's Hospital, \\ Guangdong Academy of Medical Sciences, Guangzhou 510080, China
}

Correspondence should be addressed to Jun Liang; guiandlong@163.com, Weihong Sha; shaweihong@gdph.org.cn, and Hao Chen; chenhao@gdph.org.cn

Received 13 May 2020; Revised 3 August 2020; Accepted 13 August 2020; Published 9 September 2020

Academic Editor: Toshimi Chiba

Copyright (C) 2020 Chen Zhang et al. This is an open access article distributed under the Creative Commons Attribution License, which permits unrestricted use, distribution, and reproduction in any medium, provided the original work is properly cited.

Background and Aims. Treatment options for radiation-induced intestinal injury (RIII) are limited. Crocetin has been demonstrated to exert antioxidant, antiapoptotic, and anti-inflammatory effects on various diseases. Here, we investigate the effects of crocetin on RIII in vitro. Materials and Method. IEC-6 cells exposed to $10 \mathrm{~Gy}$ of radiation were treated with different doses of crocetin $(0,0.1,1,10$, and $100 \mu \mathrm{M})$, and cell viability was assessed by CCK- 8 . The levels of superoxide dismutase (SOD), catalase (CAT), glutathione peroxidase (GPx), malondialdehyde (MDA), myeloperoxidase (MPO), tumor necrosis factor- $\alpha$ (TNF- $\alpha$ ), interleukin-1 $\beta$ (IL-1 $\beta)$, and interferon- $\gamma$ (IFN- $\gamma$ ) in culture supernatants were measured using colorimetric and ELISA kits, respectively. Cellular apoptosis was evaluated by Annexin V/PI double staining. Results. Crocetin dosedependently improved the survival of irradiated IEC-6 cells with the optimal dose of $10 \mu \mathrm{M}$, as indicated by the reduction of cellular apoptosis, decreased levels of MDA, MPO, and proinflammatory cytokines (TNF- $\alpha$, IL- $1 \beta$, and IFN- $\gamma$ ), and increased activities of antioxidative enzymes (SOD, CAT, and GPx). Conclusion. Our findings demonstrated that crocetin alleviated radiation-induced injury in intestinal epithelial cells, offering a promising agent for radioprotection.

\section{Introduction}

Radiation-induced intestinal injury (RIII) is a common complication of radiation therapy in patients with abdominal or pelvic malignancies, which seriously affects the quality of life and even leads to substantial mortality $[1,2]$. Exposure of the small intestine to radiation may produce a large amount of free radicals and epithelial cell apoptosis, which cause impaired barrier function, followed by inflammatory response and even septicemia $[3,4]$. Although RIII seriously affects the efficacy of abdominopelvic radiotherapy, there are no thera- peutic agents available to attenuate the intestinal toxicity of radiation [5].

Radioprotectors targeting oxidative damage and inflammatory reaction have been studied for decades with limited success, because of either the limited protective effect or inevitable toxicity [6]. In addition, previous studies have showed that some radioprotective agents had the risk of tumorigenesis, hampering their clinical application $[6,7]$.

Crocetin, an active constituent of saffron (Crocus sativus L) stigma, belongs to the large family of carotenoids [8]. Accumulated evidences have demonstrated that crocetin exerted 
beneficial effects on injured tissue $[9,10]$ and tumor cells $[11,12]$. It has been reported that crocetin attenuated TNBS-induced colitis in mice by reducing inflammatory cytokines and lipid peroxidation [13]. A previous study has also proved that crocetin treatment protected against burninduced intestinal injury via inhibiting oxidative stress and inflammatory response [14]. Additionally, crocetin could inhibit the growth and metastasis of tumor cells both in vitro and in vivo [15-17]. However, the potential role of crocetin on RIII has not been reported. In this study, we aimed to investigate whether and how crocetin protected against RIII.

\section{Materials and Methods}

2.1. Cell Culture. Rat intestinal epithelial IEC-6 cells were obtained from the American Type Culture Collection (ATCC, Manassas, VA, USA). Cells were maintained in high-glucose Dulbecco's modified Eagle's medium (Sigma, St Louis, MO, USA; no. D5796) with 10\% fetal bovine serum, 1\% penicillin/streptomycin, and $0.1 \mathrm{U} / \mathrm{mL}$ recombinant human insulin at $37^{\circ} \mathrm{C}$ in a humidified atmosphere containing $5 \% \mathrm{CO}_{2}$. The culture medium was changed every 2 or $3 \mathrm{~d}$. The cells were passaged as they grew to $70 \sim 80 \%$ confluence, and cells before $20^{\text {th }}$ passages were used for the experiments.

2.2. Irradiation and Crocetin Treatment. The radiation procedure was performed according to our previously described protocols [4]. Briefly, IEC-6 cells were exposed to $10 \mathrm{~Gy}$ doses of radiation using a linear accelerator (Siemens PRIMUS) at a dose-rate of $300 \mathrm{cGy} / \mathrm{min}$. IEC-6 cells were seeded into 96well plates at a density of $1 \times 10^{4}$ cells/well and grown to $70 \% \sim 80 \%$ confluence prior to experiment. After $10 \mathrm{~Gy}$ radiation, IEC-6 cells were replaced with serum-free DMEM-F12 medium and subsequently treated with different doses of crocetin $(0,0.1,1,10$, and $100 \mu \mathrm{M}, \mathrm{MP}$ Biomedicals, Santa Ana, CA, USA; CAS no.: 27876-94-4), then incubated for $24 \mathrm{~h}$ at $37^{\circ} \mathrm{C}$. After $24 \mathrm{~h}$ incubation, the culture medium was collected for biochemical assay and ELISA and then replaced with new fresh serum-free medium for subsequent condition of IEC- 6 cells. To determine the most effective concentration of crocetin in the following experiments, cell viability was assessed daily for the next 7 days after radiation. Further studies were performed at the most effective concentration to improve cell viability.

2.3. Cell Viability Assay. The viability of IEC-6 cells was assessed by CCK-8 assay, and all the steps followed the manufacturer's instruction (Dojindo Laboratories, Kumamoto, Japan; no. CK04). IEC-6 cells were cultured in 96-well plates with a density of $1 \times 10^{4}$ cells/well for $24 \mathrm{~h}$. After $10 \mathrm{~Gy}$ radiation and treatment with different doses of crocetin for $24 \mathrm{~h}$, $10 \mu \mathrm{L}$ of CCK- 8 was added to each well and for incubation for another $1 \mathrm{~h}$ at $37^{\circ} \mathrm{C}$. Cell viability was measured daily for 7 consecutive days after radiation. Absorbance of each well was determined at $450 \mathrm{~nm}$ using a Multiskan Spectrum (Thermo Fisher, CA, USA). The experiment was independently repeated at least three times.

2.4. Biochemical Measurements. Malondialdehyde (MDA, Beyotime Institute of Biotechnology, Shanghai, China; no.
S0131) levels, superoxide dismutase (SOD, Abcam, Cambridge, MA, USA; no. ab65354) activities, catalase (CAT, Sigma, St. Louis, MO, USA; no. CAT100) activities, glutathione peroxidase (GPx, Beyotime Institute of Biotechnology, Shanghai, China; no. S0056) levels, and myeloperoxidase (MPO, Abcam, Cambridge, MA, USA; no. ab105136) activities in the cell culture supernatants were measured at 1, 3, 5, and $7 \mathrm{~d}$ after radiation using commercial assay kits, respectively, according to the manufacturer's protocols.

2.5. Cell Apoptosis Assay. Cell apoptosis was detected at 1, 3, 5, and 7 days after radiation using Annexin V-FITC/PI Apoptosis Detection Kit (BD Biosciences, San Diego, CA, USA) based on our previously described procedures [18]. Briefly, IEC- 6 cells were plated in 6-well plates at a concentration of $1 \times 10^{5}$ cells/well. The cells in all groups were incubated and then harvested at $1,3,5$, and $7 \mathrm{~d}$ after treatment of crocetin, washed with PBS twice, resuspended in binding buffer, and stained with Annexin V and propidium iodide (PI) for $10 \mathrm{~min}$ at room temperature in the dark. Annexin V fluorescence was measured using a flow cytometer (BD Biosciences), and the membrane integrity of the cells was simultaneously assessed by the PI exclusion method.

2.6. Cytokine Assay. Proinflammatory cytokines tumor necrosis factor- $\alpha$ (TNF- $\alpha$, R\&D Systems, Minneapolis, MN, USA; no. PMTA00B), interleukin- $\beta$ (IL-1 $\beta$, RayBiotech, Peachtree Corners, GA, USA; no. ELM-IL1b-1), and interferon- $\gamma$ (IFN$\gamma, \mathrm{R} \& D$ Systems, Minneapolis, MN, USA; no. PMIF00) levels were obtained from the cell culture supernatants at $1,3,5$, and 7 days after radiation and were measured using ELISA kits according to the manufacturer's instructions.

2.7. Statistical Analysis. The differences of all measured parameters among groups were analyzed by one-way analysis of variance followed by Student-Newman-Keuls- (SNK-) q test and between two groups by Student $t$-test. All analyses were performed with SPSS statistics package (IBM SPSS, Chicago, IL, USA). Data were considered statistically significant for $P<0.05$.

\section{Results}

3.1. Crocetin Improved the Survival of Irradiated IEC-6 Cells. To evaluate the therapeutic mechanisms of crocetin in radiation-induced intestinal injury (RIII), we established in vitro experimental systems (Figure 1). To determine the optimal concentration of crocetin on irradiated IEC- 6 cells, the cell viability of each group was tested by CCK- 8 assay. The cell viability of IEC- 6 cells was significantly decreased after radiation (Figure 2(b)), whereas treatment with crocetin at concentrations of $0.1 \mu \mathrm{M}, 1 \mu \mathrm{M}$, and $10 \mu \mathrm{M}$ improved the survival of irradiated IEC- 6 cells in a dose-dependent manner with the maximal effect achieved at $10 \mu \mathrm{M}$ (Figures $2(\mathrm{a})$ and $2(\mathrm{~b}))$. In contrast, $100 \mu \mathrm{M}$ of crocetin showed a decrease on the cell viability of irradiated IEC- 6 cells compared to that of the irradiated group (Figures 2(a) and 2(b)). According to the results, $10 \mu \mathrm{M}$ was the most effective dose of crocetin to improve the viability of irradiated IEC-6 cells, which was used for subsequent experiments. 


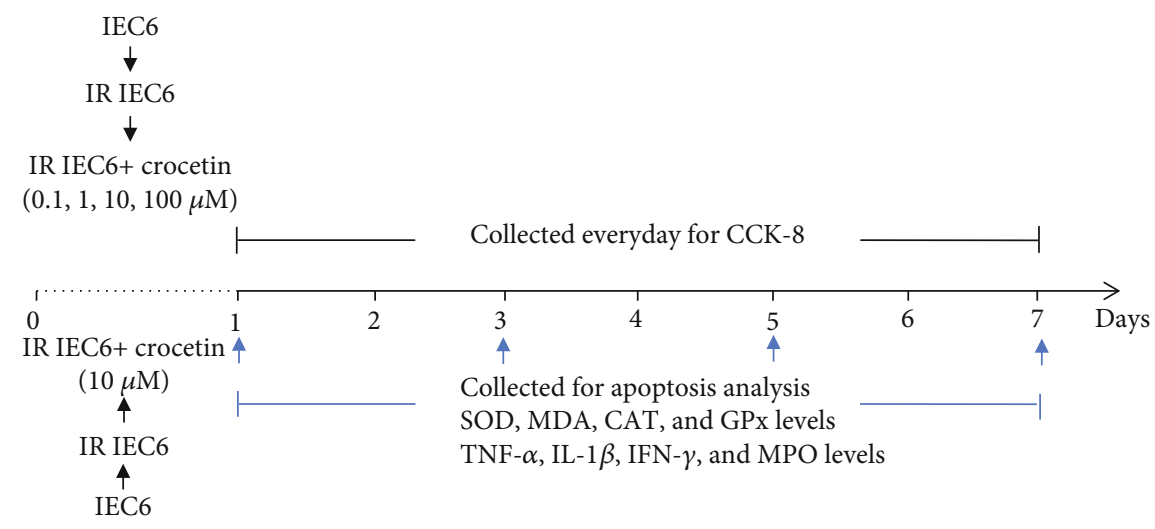

Figure 1: In vitro experiment design. IEC-6 cells were exposed to 10Gy of radiation, followed by treatment of different doses of crocetin $(0.1 \mu \mathrm{M}, 1 \mu \mathrm{M}, 10 \mu \mathrm{M}$, and $100 \mu \mathrm{M})$ for $24 \mathrm{~h}$, and culture supernatants were collected for CCK-8 assay from day 1 to day 7 after radiation. Apoptosis was detected on days $1,3,5$, and 7 after radiation. The levels of SOD, CAT, GPx, MDA, MPO, TNF- $\alpha$, IL- $1 \beta$, and IFN- $\gamma$ in culture supernatants were measured on days 1, 3, 5, and 7 after radiation. SOD: superoxide dismutase; CAT: catalase; GPx: glutathione peroxidase; MDA: malondialdehyde; MPO: myeloperoxidase; TNF- $\alpha$ : tumor necrosis factor- $\alpha$, IL- $1 \beta$ : interleukin- $1 \beta$, IFN- $\gamma$ : interferon- $\gamma$; IR: irradiation group.

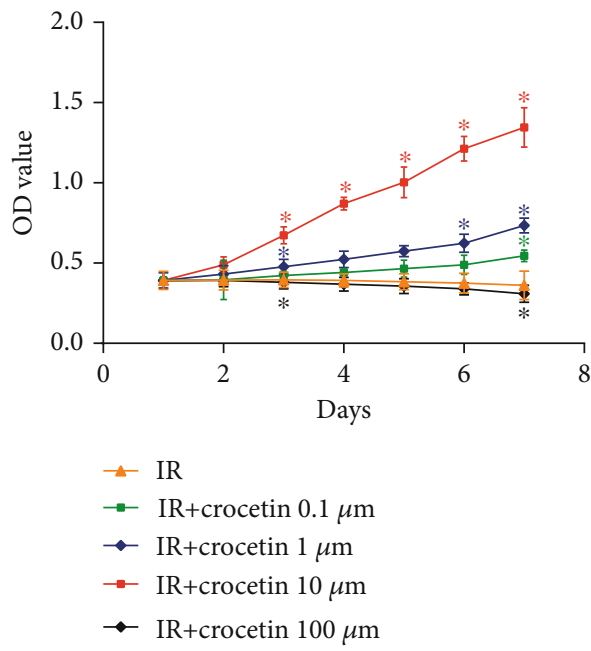

(a)

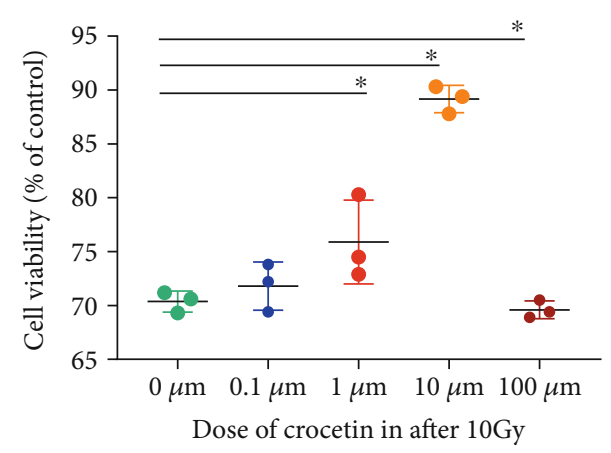

(b)

FIGURE 2: Crocetin improved the survival of irradiated IEC-6 cells in a dose-dependent manner $(0.1 \mu \mathrm{M}, 1 \mu \mathrm{M}$, and $10 \mu \mathrm{M})$. IEC-6 cells were treated with different doses of crocetin $(0.1 \mu \mathrm{M}, 1 \mu \mathrm{M}, 10 \mu \mathrm{M}$, and $100 \mu \mathrm{M})$ after exposure of $10 \mathrm{~Gy}$ radiation. (a) Cell viability was detected by CCK-8 from day 1 to day 7 after radiation. (b) Cell viability was detected by CCK- 8 on day 3 after radiation. Data represent mean \pm SD of three independent experiments. * represents $P<0.05$ as compared to the IR group. IR group: irradiation group.

\subsection{Crocetin Attenuated Oxidative Stress in Irradiated IEC-6} Cells. To investigate the effect of crocetin on oxidative stress, we examined the levels of SOD, GPx, CAT, and MDA in culture supernatants by colorimetric assays. While radiation led to increased level of MDA, this increase was alleviated by crocetin (Figure 3(b), $P<0.05$ ). Conversely, treatment of irradiated IEC-6 cells with crocetin significantly elevated the activities of endogenous antioxidant enzymes (SOD, GPx, and CAT), compared to the irradiated group (Figures 3(a), $3(\mathrm{c})$, and $3(\mathrm{~d}), P<0.05)$. These data suggested that crocetin exerted an antioxidant effect in irradiated IEC- 6 cells.

3.3. Crocetin Ameliorated Apoptosis in Irradiated IEC-6 Cells. We further evaluated the effect of crocetin on apoptosis of irradiated IEC-6 cells by Annexin V/PI double staining. The percentage of apoptotic cells increased after radiation compared to the control group (Figure 4(b), $P<0.05$ ), whereas crocetin dramatically reduced the apoptosis of irradiated IEC- 6 cells on day 3 and day $5(P<0.05)$, with less effects on day 7 (Figures 4(a)-4(c)). These results indicated that crocetin reduced radiation-induced intestinal epithelial apoptosis.

3.4. Crocetin Inhibited Inflammation in Irradiated IEC-6 Cells. To explore the effect of crocetin on inflammatory response in irradiated IEC-6 cells, the levels of proinflammatory cytokines in culture supernatants were assessed. Exposure to radiation remarkably increased the levels of TNF- $\alpha$, IL- $1 \beta$, and IFN $-\gamma$, while administration of crocetin dramatically 


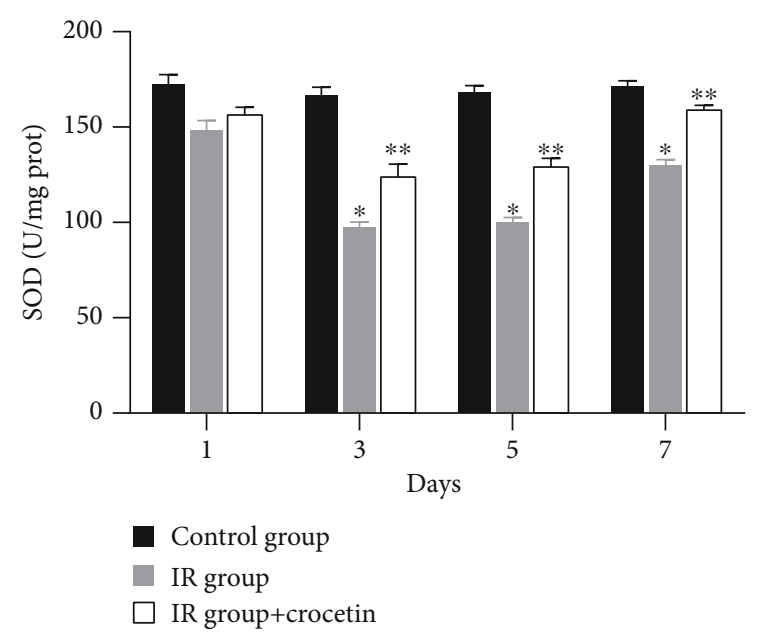

(a)

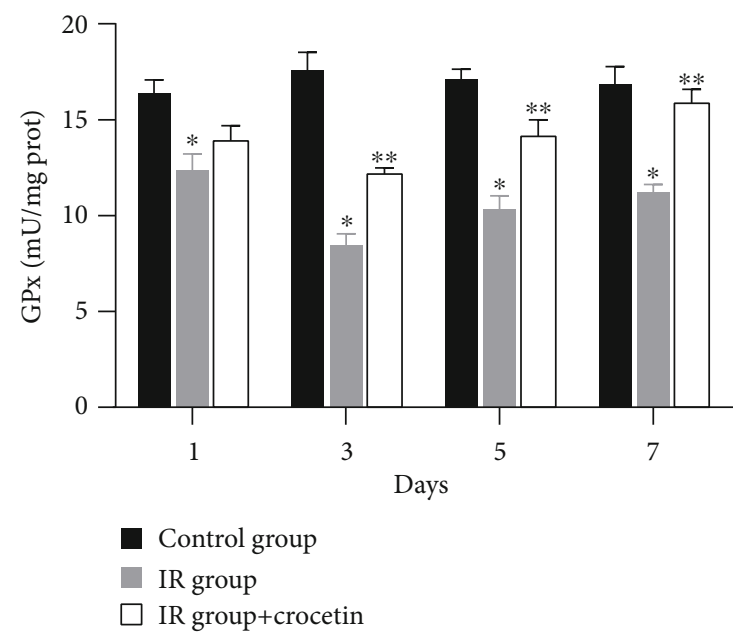

(c)

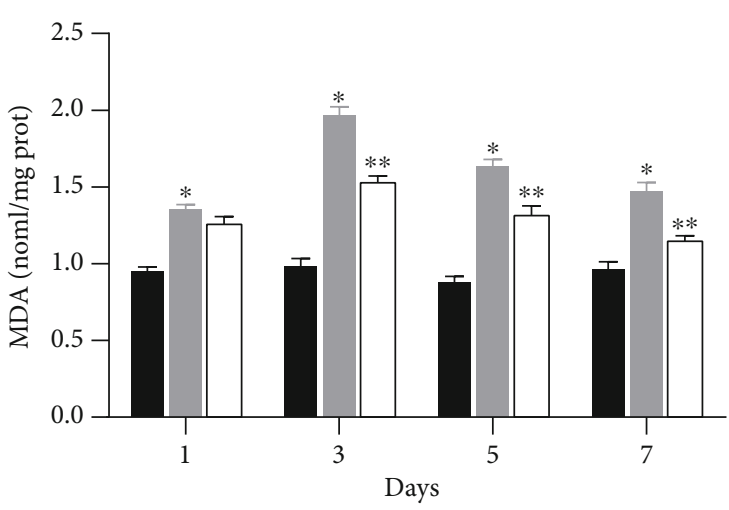

Control group
IR group
$\square$ IR group+crocetin

(b)

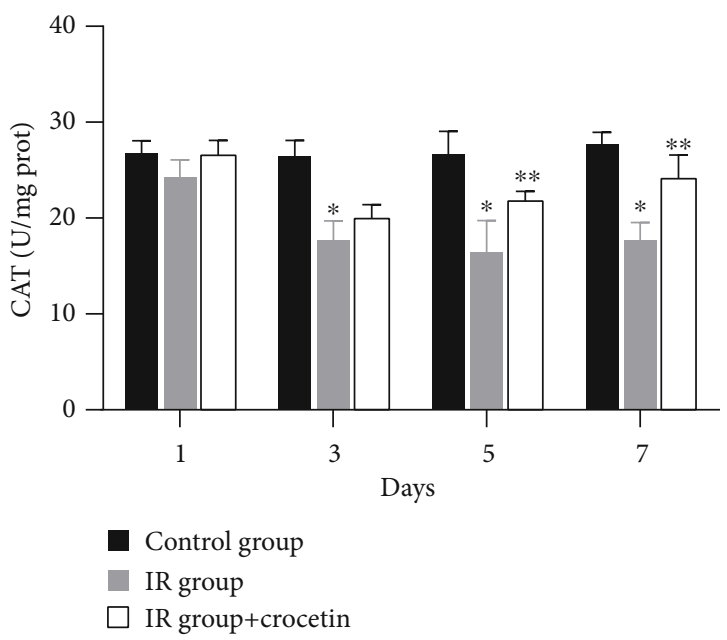

(d)

Figure 3: Crocetin attenuated oxidative stress in irradiated IEC-6 cells. The activities of (a) SOD, (b) MDA, (c) GPx, and (d) CAT in cell culture supernatants were detected on days $1,3,5$, and 7 after radiation. Data were expressed as mean \pm SD of three independent experiments. $* P<0.05$ compared to the control group, $* * P<0.05$ compared to the IR group. SOD: superoxide dismutase; MDA: malondialdehyde; GPx: glutathione peroxidase; CAT: catalase; IR group: irradiation group, IEC-6 cells exposed to $10 \mathrm{G}$ y of radiation. Control group: IEC-6 cells without irradiation or crocetin.

decreased this effect (Figures 5(a)-5(c), $P<0.05$ ). Consistent with the results of proinflammatory cytokines, crocetin significantly suppressed MPO activity (Figure 5(d), $P<0.05$ ), suggesting crocetin attenuated radiation-induced inflammation in IEC-6 cells.

\section{Discussion}

Though agents ameliorating radiation-induced damage by reducing oxidants stress and inflammation may exert protective effects against RIII, the potential toxicity and tumorigenicity must be addressed before their clinical application [6]. In contrast, crocetin could be an alternative radioprotector for RIII with low toxicity $[19,20]$ and antitumor properties $[11,21]$. In our study, we demonstrated the protective effects of crocetin against radiation-induced injury in intestinal epithelial cells and the underlying mechanisms could be attributed to inhibition of oxidative stress, cellular apoptosis, and inflammatory response, suggesting a safe and effective strategy for RIII.

There are some important discoveries in our work. First, the protective effects of crocetin in different concentrations on irradiated IEC- 6 cells were investigated. In this study, we demonstrated that lower concentrations $(0.1 \mu \mathrm{M}, 1 \mu \mathrm{M}$, and $10 \mu \mathrm{M})$ of crocetin improved the survival of irradiated IEC-6 cells in a dose-dependent manner, showing the most pronounced effect at the dose of $10 \mu \mathrm{M}$. Consistent with our findings, Yoshino et al. found that crocetin at $1 \sim 10 \mu \mathrm{M}$ protected HT22 cells against $\mathrm{A} \beta_{1-42}$-induced neuronal cell death [22]. Conversely, it was reported previously that high doses of crocetin exerted cytotoxic effects on healthy monocytes and Alzheimer's disease monocytes [23]. Our study also found that $100 \mu \mathrm{M}$ of crocetin decreased cell viability whereas no cytotoxicity was observed at $0.1 \sim 10 \mu \mathrm{M}$, suggesting the safe concentration of crocetin should be lower than $100 \mu \mathrm{M}$. These findings suggested that $10 \mu \mathrm{M}$ was relatively 


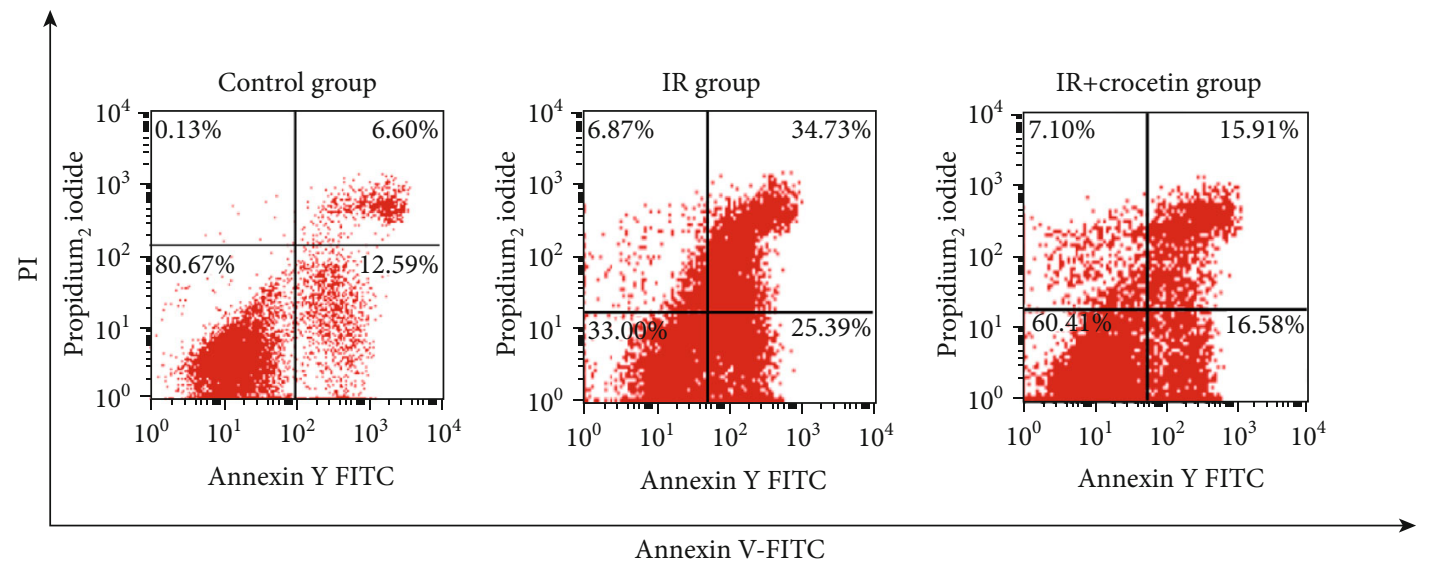

(a)

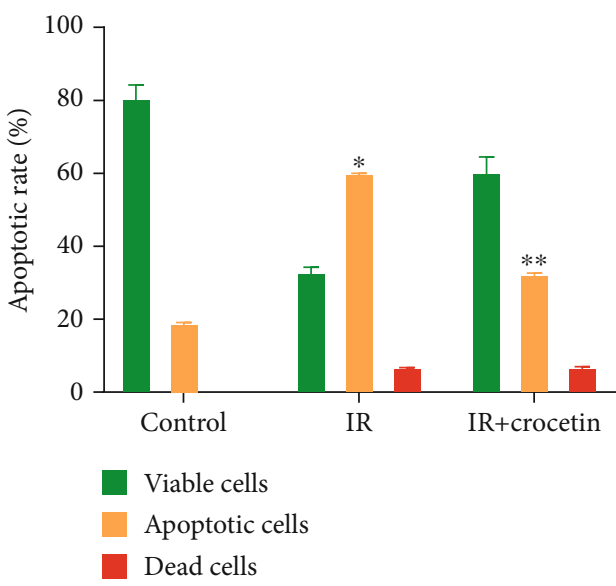

(b)

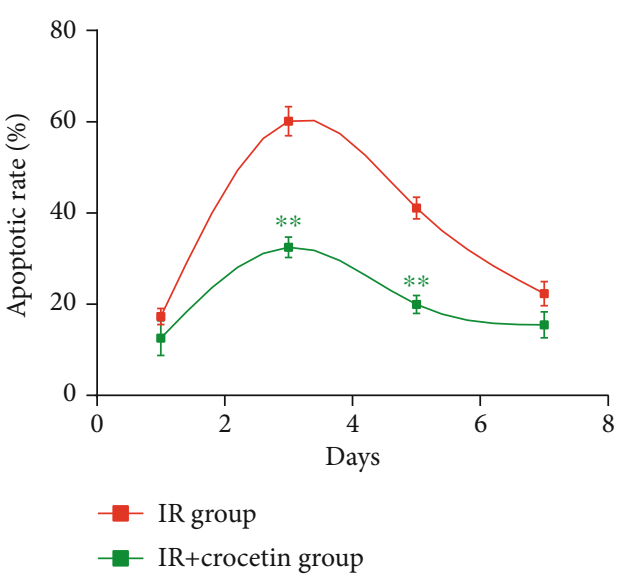

(c)

FIgURE 4: Crocetin ameliorated apoptosis in irradiated IEC-6 cells. (a) Apoptosis of IEC-6 cells was detected by flow cytometry after Annexin V/PI staining 3 days after radiation. The left upper quadrant contains necrotic cells (\%); the upper right quadrant contains late apoptotic cells (\%); the lower left quadrant contains live cells (\%); and the lower right quadrant contains early apoptotic cells (\%). (b) The percentage of total apoptotic cells and dead cells was calculated on day 3 after radiation. (c) Apoptotic ratio of IEC-6 cells was detected by Annexin V/PI double staining at $1,3,5$, and 7 days after radiation. Data were expressed as mean \pm SD of three independent experiments. $* P<0.05$ compared to the control group, $* * P<0.05$ compared to the IR group. IR group: irradiation group, IEC- 6 cells exposed to 10 Gy of radiation. Control group: IEC-6 cells without irradiation or crocetin.

a safe and effective dose of crocetin to protect irradiated IEC6 cells.

Second, our study demonstrated the mechanisms of crocetin on RIII. Previous studies showed that crocetin exerted beneficial effects on tissue regeneration by reducing oxidative stress, inhibiting cellular apoptosis, and attenuating inflammatory response [24-26]. Recently, a study further investigated that crocetin protected ultraviolet $\mathrm{A}$ radiation-induced skin damage by reducing oxygen species production and cellular apoptosis [27]. Similar with these studies, we observed that crocetin inhibited oxidative stress, the occurrence of apoptosis, and inflammation in irradiated IEC-6 cells, suggesting crocetin could attenuate intestinal toxicity induced by radiation.

Though some substances have shown variable degrees of radioprotective properties, the application of most agents is hindered by toxicity and narrow therapeutic time windows [28]. Crocetin has been reported to treat a wide range of diseases with low toxicity $[19,20]$. Milajerdi et al. suggested that $\mathrm{LD}_{50}$ values of saffron stigma extracts containing croce- tin could be very higher than the therapeutic dose [29]. A clinical study also reported that no adverse changes in volunteers were observed after crocetin was administrated at the dose of $37.5 \mathrm{mg} / \mathrm{d}$ for 4 weeks [30]. Moreover, crocetin could inhibit the proliferation and invasion of various tumor cells including intestinal cancer [31]. Kim et al. have demonstrated that crocetin could increase the death of HCT-116 colorectal cancer cells [11]. Ray et al. have also demonstrated that crocetin could induce p53-mediated cell death by p73mediated FAS-FADD-caspase-8 activation and BID cleavage in colorectal cancer cells [32]. As RIII commonly occurs in patients with abdominopelvic malignancies receiving local radiation therapy, crocetin represents a promising therapy to attenuate radiation-induced injury of intestine and, at the same time, inhibit tumor growth. However, the possible optimal doses in vivo still need to be further studied.

This study has potential limitations. First, the effect of crocetin on tumor cell lines after radiation was not studied because previous studies have reported the antitumor effects 


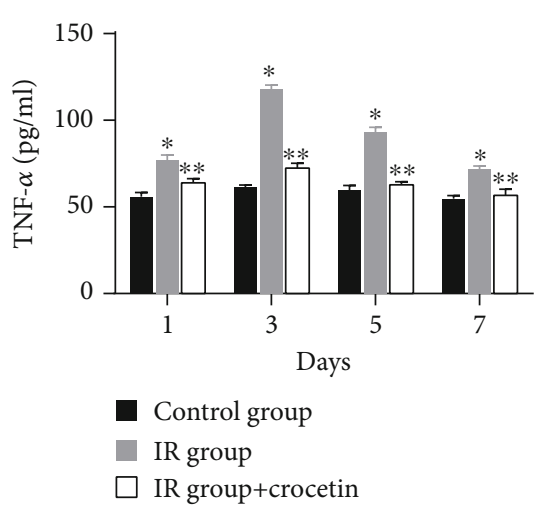

(a)

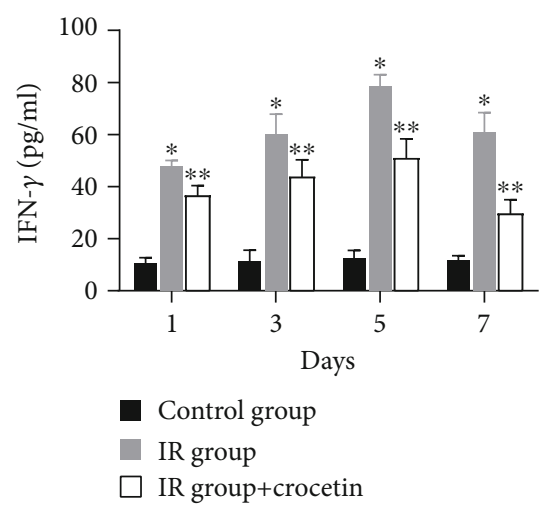

(c)

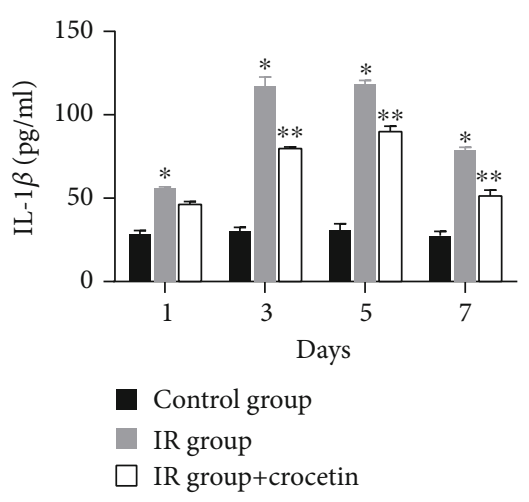

(b)

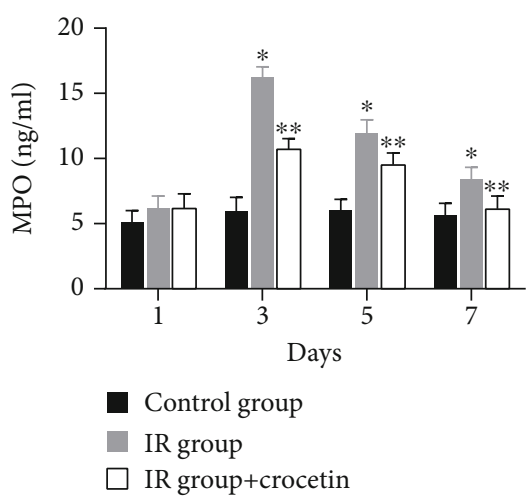

(d)

FIGURE 5: Crocetin inhibited inflammatory response in irradiated IEC-6 cells. The levels of (a) TNF- $\alpha$, (b) IL-1 $\beta$, (c) IFN- $\gamma$, and (d) MPO in cell culture supernatants were measured on days $1,3,5$, and 7 after radiation. Data were expressed as mean \pm SD of three independent experiments. $* P<0.05$ compared to the control group, $* * P<0.05$ compared to the IR group. TNF- $\alpha$ : tumor necrosis factor- $\alpha$, IL- $1 \beta$ : interleukin- $1 \beta$, IFN- $\gamma$ : interferon- $\gamma$; MPO: myeloperoxidase; IR group: irradiation group, IEC-6 cells exposed to 10 Gy of radiation. Control group: IEC-6 cells without irradiation or crocetin.

of crocetin on various tumor cells [11, 31]. Second, the solubility and the bioavailability of crocetin require optimization before being used as an effective radioprotective agent [12]. This problem may be solved with cyclodextrins or similar molecules. For example, Wong et al. suggested that crocetin- $\gamma$-cyclodextrin inclusion complex could enhance the solubility, bioavailability, and applicability of crocetin [33]. Puglia et al. showed that solid lipid nanoparticles containing crocetin improved its solubility, stability, and pharmacokinetic properties, offering an appropriated approach to resolve this issue [34]. Third, this is a study in cell model only providing preclinical clues for radioprotection of crocetin; more studies are needed to demonstrate its effects on RIII in animal models and further in clinical studies.

\section{Conclusions}

In conclusion, the present study suggests that crocetin could be an attractive agent for RIII not only attenuating intestinal injury induced by radiation via inhibiting oxidative stress, cellular apoptosis, and inflammatory response but also improving the efficacy of cancer cure with potential antitumor effects.

\section{Data Availability}

The data used to support the findings of this study are available from the corresponding author upon reasonable request.

\section{Conflicts of Interest}

The authors declare that they have no potential conflicts of interest.

\section{Authors' Contributions}

Chen Zhang, Kequan Chen, Jinghua Wang, Zhongwen Zheng, and Yujun Luo contributed equally to this work.

\section{Acknowledgments}

This work was supported by the National Natural Science Foundation of China (Grant Nos. 81300279 and 81741067), the Guangdong Province Natural Science Foundation (Grant Nos. 2016A030313815 and 2017A030313464), the Science and Technology Planning Project of Guangdong Province (Grant No. 2015A020212029), the Science and Technology Program of Guangzhou (Grant Nos. 201707010419 and 
201804010050), the Medical Scientific Research Foundation of Guangdong Province (Grant Nos. A2018343 and A2019063), the Fundamental Research Funds for the Central Universities (Grant No. D2180600), the Scientific Research Project of Traditional Chinese Medicine Bureau of Guangdong Province (Grant Nos. 20201009 and 20201010), and the High-Level Hospital Construction Project (Grant Nos. DFJH201803, KJ012019099, and DFJH201923).

\section{References}

[1] Y. Li, J. Dong, H. Xiao et al., "Gut commensal derived-valeric acid protects against radiation injuries," Gut Microbes, vol. 11, no. 4, pp. 789-806, 2020.

[2] J.-S. Kim, M. Yang, C.-G. Lee, S.-D. Kim, J.-K. Kim, and K. Yang, "In vitro and in vivo protective effects of granulocyte colony-stimulating factor against radiation-induced intestinal injury," Archives of Pharmacal Research, vol. 36, no. 10, pp. 1252-1261, 2013.

[3] L. Moussa, B. Usunier, C. Demarquay et al., "Bowel radiation injury: complexity of the pathophysiology and promises of cell and tissue engineering," Cell Transplantation, vol. 25, no. 10, pp. 1723-1746, 2016.

[4] H. Chen, X. H. Min, Q. Y. Wang et al., "Pre-activation of mesenchymal stem cells with TNF- $\alpha$, IL- $1 \beta$ and nitric oxide enhances its paracrine effects on radiation-induced intestinal injury," Scientific Reports, vol. 5, no. 1, p. 8718, 2015.

[5] A. K. Shadad, F. J. Sullivan, J. D. Martin, and L. J. Egan, "Gastrointestinal radiation injury: prevention and treatment," World Journal of Gastroenterology, vol. 19, no. 2, pp. 199208, 2013.

[6] C. N. Andreassen, C. Grau, and J. C. Lindegaard, "Chemical radioprotection: a critical review of amifostine as a cytoprotector in radiotherapy," Seminars in Radiation Oncology, vol. 13, no. 1, pp. 62-72, 2003.

[7] M. Hauerjensen, J. Wang, and J. Denham, "Bowel injury: current and evolving management strategies," Seminars in Radiation Oncology, vol. 13, no. 3, pp. 358-371, 2003.

[8] M. Giaccio, "Crocetin from saffron: an active component of an ancient spice," Critical Reviews in Food Science and Nutrition, vol. 44, no. 3, pp. 155-172, 2004.

[9] F. Ishizuka, M. Shimazawa, N. Umigai et al., "Crocetin, a carotenoid derivative, inhibits retinal ischemic damage in mice," European Journal of Pharmacology, vol. 703, no. 1-3, pp. 1-10, 2013.

[10] Y. Wang, J. Sun, C. Liu, and C. Fang, "Protective effects of crocetin pretreatment on myocardial injury in an ischemia/reperfusion rat model," European Journal of Pharmacology, vol. 741, pp. 290-296, 2014.

[11] S. H. Kim, J. M. Lee, S. C. Kim, C. B. Park, and P. C. Lee, "Proposed cytotoxic mechanisms of the saffron carotenoids crocin and crocetin on cancer cell lines," Biochemistry and Cell Biology, vol. 92, no. 2, pp. 105-111, 2014.

[12] A. Colapietro, A. Mancini, A. M. D'Alessandro, and C. Festuccia, "Crocetin and crocin from saffron in cancer chemotherapy and chemoprevention," Anti-Cancer Agents in Medicinal Chemistry, vol. 19, no. 1, pp. 38-47, 2019.

[13] Z. Qian and H. A. Kazi, "Crocetin reduces TNBS-induced experimental colitis in mice by downregulation of NFkB," Saudi Journal of Gastroenterology, vol. 15, no. 3, pp. 181187, 2009.
[14] C. Zhou, W. Bai, Q. Chen et al., "Protective effect of crocetin against burn-induced intestinal injury," Journal of Surgical Research, vol. 198, no. 1, pp. 99-107, 2015.

[15] S. Z. Bathaie, R. Hoshyar, H. Miri, and M. Sadeghizadeh, "Anticancer effects of crocetin in both human adenocarcinoma gastric cancer cells and rat model of gastric cancer," Biochemistry and Cell Biology, vol. 91, no. 6, pp. 397-403, 2013.

[16] A. Dhar, S. Mehta, G. Dhar et al., "Crocetin inhibits pancreatic cancer cell proliferation and tumor progression in a xenograft mouse model," Molecular Cancer Therapeutics, vol. 8, no. 2, pp. 315-323, 2009.

[17] B. Chen, Z. H. Hou, Z. Dong, and C. D. Li, "Crocetin downregulates the proinflammatory cytokines in methylcholanthreneinduced rodent tumor model and inhibits COX-2 expression in cervical cancer cells," BioMed Research International, vol. 2015, Article ID 829513, 5 pages, 2015.

[18] H. Chen, H. Zhang, Y. Zheng et al., "Prolyl hydroxylase 2 silencing enhances the paracrine effects of mesenchymal stem cells on necrotizing enterocolitis in an NF- $\kappa \mathrm{B}$-dependent mechanism," Cell Death \& Disease, vol. 11, no. 3, p. 188, 2020.

[19] A. H. Mohamadpour, Z. Ayati, M. R. Parizadeh, O. Rajbai, and H. Hosseinzadeh, "Safety evaluation of crocin (a constituent of saffron) tablets in healthy volunteers," Iranian Journal of Basic Medical Sciences, vol. 16, no. 1, pp. 39-46, 2013.

[20] M. Hashemi and H. Hosseinzadeh, "A comprehensive review on biological activities and toxicology of crocetin," Food and Chemical Toxicology, vol. 130, pp. 44-60, 2019.

[21] C. Festuccia, A. Mancini, G. L. Gravina et al., "Antitumor effects of saffron-derived carotenoids in prostate cancer cell models," BioMed Research International, vol. 2014, Article ID 135048, 12 pages, 2014.

[22] Y. Yoshino, M. Ishisaka, N. Umigai, M. Shimazawa, K. Tsuruma, and H. Hara, "Crocetin prevents amyloid $\beta_{1-42^{-}}$ induced cell death in murine hippocampal cells," Pharmacology \& Pharmacy, vol. 5, no. 1, pp. 37-42, 2014.

[23] R. Tiribuzi, L. Crispoltoni, V. Chiurchiù et al., "Trans-crocetin improves amyloid- $\beta$ degradation in monocytes from Alzheimer's disease patients," Journal of the Neurological Sciences, vol. 372, pp. 408-412, 2017.

[24] K. N. Nam, Y. M. Park, H. J. Jung et al., "Anti-inflammatory effects of crocin and crocetin in rat brain microglial cells," European Journal of Pharmacology, vol. 648, no. 1-3, pp. 110-116, 2010.

[25] K. Niska, M. J. Santos-Martinez, M. W. Radomski, and I. Inkielewicz-Stepniak, "CuO nanoparticles induce apoptosis by impairing the antioxidant defense and detoxification systems in the mouse hippocampal HT22 cell line: protective effect of crocetin," Toxicology in Vitro, vol. 29, no. 4, pp. 663-671, 2015.

[26] T. H. Tseng, C. Y. Chu, J. M. Huang, S. J. Shiow, and C. J. Wang, "Crocetin protects against oxidative damage in rat primary hepatocytes," Cancer Letters, vol. 97, no. 1, pp. 61-67, 1995.

[27] T. Ohba, M. Ishisaka, S. Tsujii et al., "Crocetin protects ultraviolet $\mathrm{A}$-induced oxidative stress and cell death in skin in vitro and in vivo," European Journal of Pharmacology, vol. 789, pp. 244-253, 2016.

[28] M. Hauer-Jensen, J. W. Denham, and H. J. N. Andreyev, "Radiation enteropathy-pathogenesis, treatment and prevention," Nature Reviews Gastroenterology \& Hepatology, vol. 11, no. 8, pp. 470-479, 2014. 
[29] A. Milajerdi, K. Djafarian, and B. Hosseini, "The toxicity of saffron (Crocus sativus L.) and its constituents against normal and cancer cells," Journal of Nutrition \& Intermediary Metabolism, vol. 3, pp. 23-32, 2016.

[30] S. Yamashita, T. Kakinuma, N. Umigai, and T. Takara, "Safety evaluation of excessive intake of crocetin in healthy adult volunteers: - a randomized, double-blind, placebo-controlled, parallel-group comparison trial," Japanese Pharmacology and Therapeutics, vol. 46, no. 3, pp. 393-401, 2018.

[31] W. G. Gutheil, G. Reed, A. Ray, S. Anant, and A. Dhar, "Crocetin: an agent derived from saffron for prevention and therapy for cancer," Current Pharmaceutical Biotechnology, vol. 13, no. 1, pp. 173-179, 2012.

[32] P. Ray, D. Guha, J. Chakraborty et al., "Crocetin exploits p53induced death domain (PIDD) and FAS-associated death domain (FADD) proteins to induce apoptosis in colorectal cancer," Scientific Reports, vol. 6, no. 1, article 32979, 2016.

[33] K. H. Wong, Y. Xie, X. Huang et al., "Delivering crocetin across the blood-brain barrier by using $\gamma$-cyclodextrin to treat Alzheimer's disease," Scientific Reports, vol. 10, no. 1, p. 3654, 2020.

[34] C. Puglia, D. Santonocito, T. Musumeci et al., "Nanotechnological approach to increase the antioxidant and cytotoxic efficacy of crocin and crocetin," Planta Medica, vol. 85, no. 3, pp. 258-265, 2019. 